\title{
Towards Formalising Agent Argumentation over the Viability of Human Organs for Transplantation
}

\author{
Sanjay Modgil, ${ }^{1}$ Pancho Tolchinsky ${ }^{2}$, and Ulises Cortés ${ }^{2}$ \\ 1 Advanced Computation Lab, Cancer Research UK \\ ${ }^{2}$ Universitat Politècnica de Catalunya
}

\begin{abstract}
In this paper we describe a human organ selection process in which agents argue over whether a given donor's organ is viable for transplantation. This process is framed in the CARREL System; an agent-based organization designed to improve the overall transplant process. We formalize an argumentation based framework that enables CARREL agents to construct and assess arguments for and against the viability of a donor's organ for a given potential recipient. We believe that the use of argumentation has the potential to increase the number of human organs that current selection processes make available for transplantation.
\end{abstract}

\section{Introduction}

Human organ transplantation constitutes the only effective therapy for many life-threatening diseases. However, while the increasing success of transplants has led to increase in demand, the lack of a concomitant increase in donor organ availability has led to a growing disparity between supply and demand. Hence, much research has focussed on definition and implementation of policies for increasing donor availability, identification of suitable recipients for organs, and procedures to increase the chances of successful transplantation. Furthermore, the scarcity of donors has led to the creation of national and international coalitions of transplant organizations. This has resulted in requirements for managing and processing vast and complex data, and accommodation of a complex set of, in some cases conflicting, national and international regulations and protocols governing exchange of organs and tissues. Hence, in [17] an agent-based architecture - CARREL - is proposed for managing the data to be processed in carrying out recipient selection, organ and tissue allocation, ensuring adherence to legislation, and following approved protocols and preparing delivery plans.

In this paper we focus on CARREL's support for donor organ (rather than tissue) transplantation. In particular, we formalise a framework for agent argumentation over organ viability for transplantation with the aim of increasing the number of human organs that current selection processes make available for transplantation. In $\$ 2$ we briefly describe CARREL and the current organ selection and assignation process in which an agent representing the hospital in which the donor is located (the donor agent) initially identifies an organ as viable or non-viable for transplantation. If identified as non-viable, then the organ is discarded (not extracted from the potential donor) rather than being offered to agents representing potential recipients. However, this process does not account for the fact that doctors may disagree as to whether any given set of 
criteria constitute an acceptable justification for identifying an organ as viable or nonviable. For example, while a donor agent may argue that an organ is non-viable, it may well be that a recipient agent provides a stronger argument for considering the organ as viable. On the other hand, a donor agent may argue that an organ is viable, and this argument may be stronger than a recipient agent's argument for non-viability. Hence, in $\$ 2$ we describe an extension to the current CARREL architecture and a new organ selection and assignation process, so as to facilitate agent argumentation over the viability of organs. In this way, organs that ordinarily would be discarded having been deemed non-viable by the donor agent, may now be successfully transplanted to a recipient with a winning argument for viability. Organs that ordinarily would be discarded if deemed non-viable by all recipient agents, may now be successfully transplanted to a recipient whose argument for non-viable is defeated by the donor's argument for viability.

In $\$ 3$ we formalise a framework for the required agent argumentation over the viability of organs. We formalise a logic programming style approach to argument construction and describe and motivate how our formalism differs from existing logic programming style approaches [12,6]. We also define conflict based interactions between the constructed arguments and relations that additionally account for some relative valuation of the strength of arguments in order that one argument may defeat its conflicting counterpart. We then describe Dung's seminal calculus of opposition [4] for determining the preferred (winning) arguments on the basis of the ways in which they interact. Finally, 44 concludes with a discussion and programme for future work.

\section{The Carrel Institution and the Organ Selection and Assignation Process}

CARREL is an electronic institution in which the interactions among a group of agents are governed by a set of norms expressed in the ISLANDER specification language [5]. CARREL is formalized as an electronic institution; a type of dialogical system where all the interactions are compositions of message exchanges, or illocutions, structured through agent group meetings called scenes or rooms. Each agent can be associated with one or more roles, and these roles define the rooms the agent can enter and the protocols it should follow. Figure 1a) shows the CARREL institution and the hospitals $U C T_{1} \ldots U C T_{n}$ that are members of CARREL. Each $U C T x$ is modelled as an agency. The roles the different agents play in this agency are described in [3]. Here we focus on donor $(D A)$ and recipient agent $(R A)$ associated with each $U C T x$, and describe their roles in the organ selection and assignation process. In particular, fig.1a) shows the donor and recipient agents $D A_{1}$ and $R A_{1}$ for $U C T 1$, and only the recipient agents $R A_{2} \ldots R A_{n}$ for hospitals $U C T_{2} \ldots U C T_{n}$. Encoded in CARREL are sets of legislation and protocols governing the exchange of organs and tissues. These are based on two physical institutions representing examples of best practice: the OCATT (Organització CATalana de Trasplantaments) [8] and ONT (Organización Nacional de Transplantes) [9] organ transplantation organizations for Catalonia and Spain respectively. A hospital becomes a member of CARREL in order to make use of the services provided. In so doing, it commits to respecting the norms that rule the interactions inside CARREL. The current selection and assignation process begins when $D A_{1}$ detects a potential 


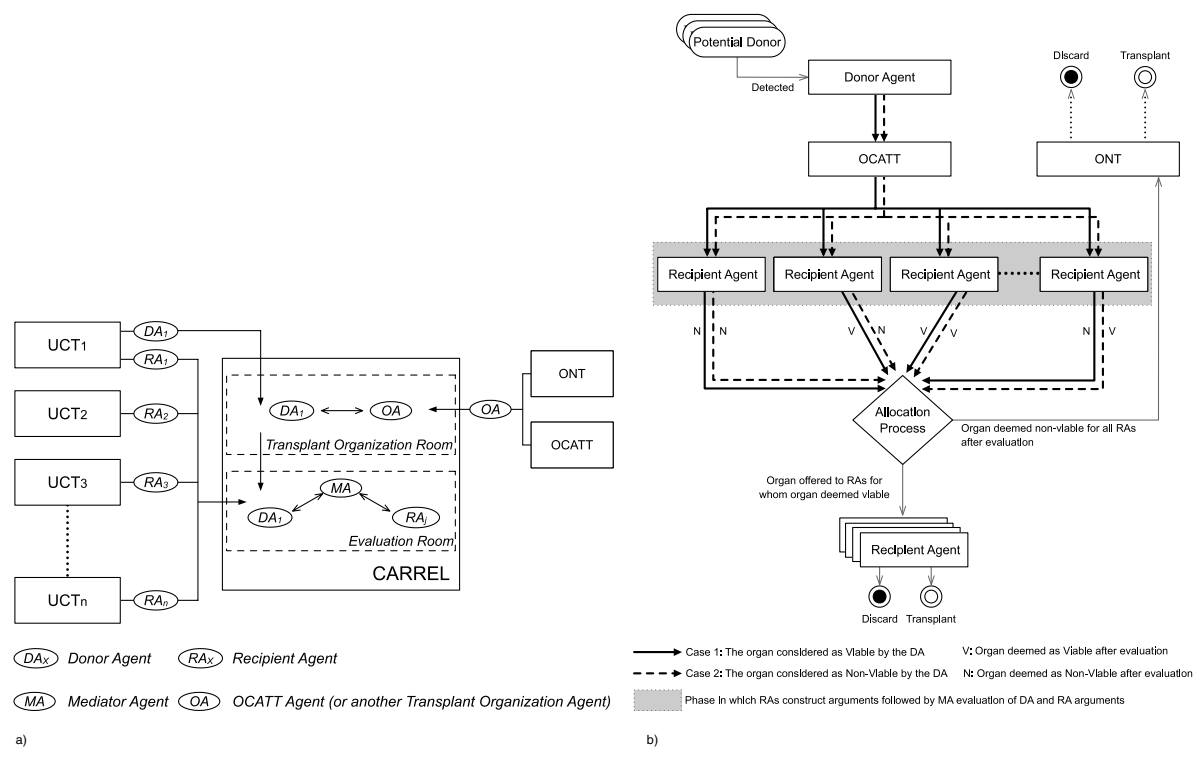

Fig. 1. a) The CARREL Institution b) Flow of the proposed human organ selection process

donor. $D A_{1}$ informs OCATT (assuming all $U C T_{i}$ are in Catalonia) only if the donor's organs are deemed viable for transplantation. Organs deemed as non-viable are discarded. OCATT then offers organs to potential recipients in a prioritised queue. Once an organ is accepted, CARREL agents are then deployed to coordinate extraction of the organ and delivery to the highest prioritised recipient that accepts the organ as viable (an organ accepted by an RA may subsequently be discarded, eg. when a surgeon deems the organ non-viable at the time of operation). However, if no potential recipients are found, then OCATT offers the organ to the ONT, and a similar process takes place, this time embracing the whole of Spain. In case of refusal, the organ is then offered to transplant organizations in Europe. If every organization fails to allocate the organ, then the organ will be discarded. Currently, in Catalonia, between 15 and $20 \%$ of livers, $20 \%$ of kidneys, $60 \%$ of hearts, $85 \%$ of lungs and $95 \%$ of pancreases are discarded [8].

We now describe a new organ selection and assignation process (illustrated in fig. 1b) that aims to decrease the number of discards and therefore reduce the disparity between supply and demand of organs. To facilitate this process, the roles of the $D A \mathrm{~s}$ and $R A \mathrm{~s}$ have been extended to include construction, sending and retrieving of arguments. A mediator agent (MA) is also defined with the role of constructing further arguments, assigning strengths to arguments and evaluating the status of interacting arguments. In addition, two new scenes or rooms are defined: a Transplant Organization Room (TOR) and an Evaluation Room (ER). Having identified a potential donor, $D A_{1}$ enters the TOR (see fig 1a) and communicates basic organ data (such as the organ type) and donor data (such as the donor's clinical history) to the $O A$ agent representing the transplant organizations (e.g., OCATT or ONT). $D A_{1}$ also sends its arguments for whether it considers the organ to be viable or non-viable to the $M A$ in the ER. The $O A$ agent 
contacts each $R A$ identified as a potential recipient on the basis of basic organ and donor data. Each contacted $R A$ then constructs its own arguments for either the viability or non-viability of the organ, and communicates these arguments to the $M A$. In the case that some $R A_{j}$ and $D A_{1}$ disagree as to the viability of the organ, MA evaluates $R A_{j}$ and $D A_{1}$ 's arguments in order to determine the winning argument, and so decide whether the organ is viable or not for $R A_{j}$. We now formalise the above described argumentation.

\section{Arguing over the Viability of Organs}

The organ assignment process illustrates the ubiquity of disagreement and conflict of opinion in the medical domain. What may be a sufficient reason for discarding an organ for some qualified professionals may not be for others. Different policies in different hospitals and regions exist, and requiring a consensus among medical professionals is not feasible. Hence, contradictory conclusions may be derived from the same set of facts. For example, suppose a donor with a smoking history of more than 20-30 packs a year and no history of chronic obstructive pulmonary disease (COPD). Some would cite a donor's smoking history as sufficient reason for labelling a donor's lung as nonviable [8]. However, there are qualified physicians that reason that the donor's lung is viable given that there is no history of COPD [7].

We propose the use of argumentation [13] to formalise the required reasoning and arbitration in the presence of conflict. Argumentation involves logic based inference of arguments followed by definition of the status of arguments on the basis of the ways in which they interact. In what follows we define the agents' inference of arguments, built from a first order logic-programming style language $\mathcal{L}$, and define evaluation of the status of arguments on the basis of conflict based interactions that additionally account for the relative strengths of arguments.

\subsection{Inference of Arguments}

A wff of $\mathcal{L}$ is an atomic first order formula or such a formula preceded by strong negation $\neg$. Let us call such formulae strong literals. An agent's knowledge base $\Delta$ consists of the union of a set $\mathcal{K}$ of ground strong literals and a set $\mathcal{R}$ of defeasible rules also written in $\mathcal{L}$. The antecedent of such a rule is built from a conjunction of strong literals and/or weak literals of the form $\sim L$, where $L$ is a strong literal and $\sim$ represents weak negation, i.e., $L$ cannot be shown to be true (negation as failure).

Definition 1. A defeasible rule is of the form:

1) $L_{1} \wedge \ldots \wedge L_{m} \Rightarrow L_{m+1}$, or

2) $L_{1} \wedge \ldots \wedge L_{m} \Rightarrow \neg R$

where $L_{i}(0 \leq i \leq m)$ is a strong or weak literal and $R$ is a rule of type 1) or 2).

An example of a rule of type 1$)$ is $p(X) \wedge \sim \neg q(X) \Rightarrow s(X)$. Note that a rule of type 2 ) with consequent $\neg R$ represents a challenge to any inference obtained by application of $R$. For instance, $r(X) \Rightarrow \neg(p(X) \wedge \sim \neg q(X) \Rightarrow s(X))$ and $t(X) \Rightarrow \neg(r(X) \Rightarrow$ $\neg(p(X) \wedge \sim \neg q(X) \Rightarrow s(X)))$. The rationale for these non-standard rules with (possibly nested) negations of rules as consequents, will be discussed later. In the following 
definition of argument inference we write $\Theta(\alpha)$ to denote the application of a substitution $\Theta=\left\{X_{1} / t_{1}, \ldots X_{n} / t_{n}\right\}$ to a first order formula or rule $\alpha$, where $X_{i}$ are the variables in $\alpha$ and $t_{i}$ are terms (constants or variables).

Definition 2. Let $\Delta=(\mathcal{K} \cup \mathcal{R})$ be an agent knowledge base and $\alpha$ denote a strong literal, or an expression of the form $R$ or $\neg R$ where $R$ is a defeasible rule. Then:

$$
-\Delta \sim \alpha \text { iff }
$$

- $\alpha \in \Delta$, or

- there exists a rule $r=L_{1} \wedge \ldots \wedge L_{n} \Rightarrow \alpha^{\prime} \in \mathcal{R}$, and a substitution $\Theta=$ $\left\{X_{1} / t_{1}, \ldots X_{n} / t_{n}\right\}$ on $r$ such that $\alpha=\Theta\left(\alpha^{\prime}\right)$, and for $i=1 \ldots n, \Delta \vdash \Theta\left(L_{i}\right)$ where each variable in $\Theta\left(L_{i}\right)$ is assumed existentially quantified

$-\Delta \sim \sim L$ iff it is not the case that $\Delta \sim L$

Definition 3. An argument based on $\Delta=(\mathcal{K} \cup \mathcal{R})$ is a tuple $(H, h)$ where:

$-H \subseteq \Delta$

$-H \sim h$

- $H$ is minimal w.r.t set inclusion $\left(\neg \exists H^{\prime} \mid H^{\prime} \subseteq H\right.$ and $\left.H^{\prime} \sim h\right)$

The above defines the standard support-claim structuring of an argument [14] in which $H$ is the support and $h$ the claim of argument $(H, h)$. A sub-argument of $(H, h)$ is of the form $\left(H^{\prime}, h^{\prime}\right)$ where $H^{\prime}$ is a subset of $H$. From hereon we assume $\mathcal{R}$ in $\Delta$ to be finite, in which case the arguments inferred from $\Delta$ will be finite (up to renaming of variables). Also, we use upper case letters to denote variables and lower case letters to denote constants. Note that by definition, each rule $R \in \mathcal{R}$ is the claim of an argument $(\{R\}, R)$, and if $\neg R$ is the consequent of a rule $R^{\prime}$ whose antecedent can be inferred from $\Delta$, then $R^{\prime}$ will be in the support of an argument with claim $\neg R\left(\left\{R^{\prime}\right\}, \neg R\right)$.

Example 1. Let $r$ be a potential recipient for the donor $d$ 's lung. Let $d_{-} p$ stand for 'donor property', $d \_o$ for 'donor organ' $s \_h$ for 'smoking history', copd for 'chronic obstructive pulmonary disease', $v$ for 'viable' and contra for 'contraindication'. Suppose DA's knowledge base $\Delta_{d}$ containing:

$$
\begin{aligned}
& d 1=d_{-} o(d, \text { lung }), \mathrm{d} 2=d_{-} p\left(d, s_{-} h\right), \mathrm{d} 3=\neg d_{-} p(d, \text { copd }), \\
& \mathrm{d} 4=d_{-} o(D, \text { lung }) \wedge d_{-} p\left(D, s_{-} h\right) \Rightarrow \text { contra }(D, \text { lung }), \\
& \mathrm{d} 5=\text { contra }(D, O) \Rightarrow \neg v(D, O)
\end{aligned}
$$

and the recipient agent's knowledge base $\Delta_{r}$ containing:

$$
\begin{aligned}
& r 1=d_{-} o(d, \text { lung }), \mathrm{r} 2=d_{-} p\left(d, s_{-} h\right), \mathrm{r} 3=\neg d_{-} p(d 1, \text { copd }), \mathrm{r} 4=\text { match }(d, r) \\
& \mathrm{r} 5=\neg d_{-} p(D, \text { copd }) \Rightarrow \neg\left(d_{-} o(D, \text { lung }) \wedge d_{-} p\left(D, s_{-} h\right) \Rightarrow \text { contra }(D, \text { lung })\right),
\end{aligned}
$$

r6 $=d_{-} o(D, O) \wedge \operatorname{match}(D, R) \wedge \sim \operatorname{contra}(R, O) \Rightarrow v(D, O)$

From $\Delta_{d}$ one can construct arguments:

- $A 1=\left(\{d 4, d 1\}, d \_o(d\right.$, lung $) \wedge d \_p\left(d, s \_h\right) \Rightarrow$ contra $(d$, lung $\left.)\right)$

- $A 2=(\{d 1, d 2, d 4\}$, contra $(d$, lung $))$

- $A 3=(\{d 1, d 2, d 4, d 5\}, \neg v(d$, lung $))$

and from $\Delta_{r}$ the arguments:

- $B 1=\left(\{r 3, r 5\}, \neg\left(d_{-} o(d\right.\right.$, lung $) \wedge d_{-} p\left(d, s_{-} h\right) \Rightarrow \operatorname{contra}(d$, lung $\left.\left.)\right)\right)$

- B2 $=(\{r 1, r 4, r 6\}, v(d$, lung $))$ 


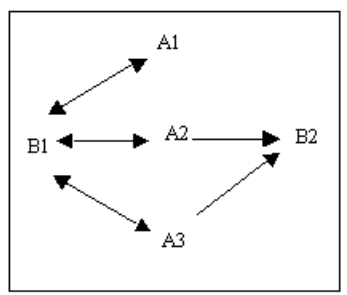

a)

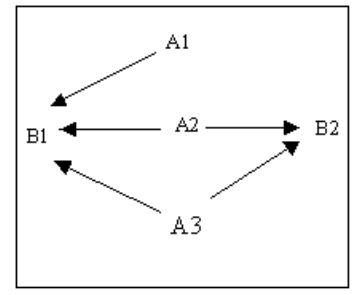

b)

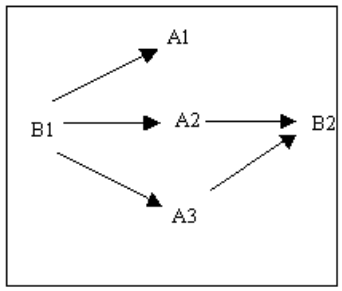

c)

Fig. 2. The arguments' defeat relations for the smoking history example

Notice that we do not formulate $r 5$ as $d_{\_} o(D$, lung $) \wedge d_{-} p\left(D, s_{\_} h\right) \wedge \neg d_{-} p(D, c o p d)$ $\Rightarrow \neg \operatorname{contra}(D$, lung $)$, as this would result in a RA's argument for $\neg$ contra $(d$, lung $)$ which would challenge any DA's argument for contra(d, lung), and not just DA's arguments constructed on the basis of the donor's smoking history. That is, $B 1$ represents a possible challenge to any argument for contra(d, lung) constructed using $d 4$.

\subsection{Defining Defeat among Arguments and Evaluating the Status of Arguments}

We now define the binary relation of defeat on pairs of conflicting arguments. This relation also accounts for a relative valuation of arguments encoded as a partial ordering.

Definition 4. Let Args be the set of arguments $\left\{\left(H_{1}, h_{1}\right) \ldots\left(H_{n}, h_{n}\right)\right\}$ inferred from a knowledge base $\Delta$, and $\preceq$ a partial ordering on Args. Then Defeat $\subseteq$ (Args $\times$ Args $)$ where $\left((H, h),\left(H^{\prime}, h^{\prime}\right)\right) \in$ De feat iff there exists a sub-argument $(G, g)$ of $(H, h)$ and a sub-argument $\left(G^{\prime}, g^{\prime}\right)$ of $\left(H^{\prime}, h^{\prime}\right)$ such that:

- there exists a $L_{1} \wedge \ldots \wedge L_{n} \Rightarrow \alpha \in G^{\prime}$ such that for some $i, L_{i}=\sim g$. In this case we say that $(H, h)$ undercut defeats $\left(H^{\prime}, h^{\prime}\right)$

$-g \equiv \neg g^{\prime}$, and it is not the case that $\left(H^{\prime}, h^{\prime}\right) \succ(H, h)$ or that $\left(H^{\prime}, h^{\prime}\right)$ undercut defeats $(H, h)$. In this case we say that $(H, h)$ rebut defeats $\left(H^{\prime}, h^{\prime}\right)$

Note that rebut defeats can be symmetrical in the absence of a partial order on Args, or when the rebutting arguments have equal strength. Note also the special case of rebut defeats between arguments with claims $R$ and $\neg R$ where $R$ is a defeasible rule. These are related to the notion of a Pollock undercut defeat [11]: $A 1$ (with claim $\neg R$ ) denies the relation between premises and conclusion of $R$ used in argument $A 2$, and thus undercut defeats $A 2$ if $A 1 \succeq A 2$. However, if $A 2 \succ A 1$ then neither argument defeats each other and so both can inappropriately co-exist in a conflict free set of arguments. One solution is to say that $A 1$ 's undercut defeat on $A 2$ always succeeds (irrespective of their relative strength). This is the approach that is effectively adopted in other logic programming based approaches (e.g. [12]) whereby a Pollock undercut is simulated by an argument $A 1$ with claim $\neg$ applicable_$R(X)$ undercut defeating $A 2$ by disproving the non-provability assumption $\sim \neg$ applicable $R(X)$ in the antecedent of $A 2$ 's rule $R$.

Our approach is to change the nature of the attack from an undercut to a rebut, so as to allow for the rule $R$ to 'repel' and indeed defeat it's attacker $\neg R$. It is partly 
for this reason that rules and negations of rules can be the claims of arguments in our formalism 1 . Example 1 illustrates our motivation. We not only want that the recipient's argument $B 1$ can defeat a donor argument's use of $d 4$, but also that an argument using $d 4$ can defeat $B 1$. Hence, we do not include $\sim \neg d_{-} p(D, c o p d)$ as an exception in the antecedent of d4 as this would preclude construction of the DA's argument $A 3$ for nonviability and its subsequent possible evaluation as a wining argument over $B 2$.

Referring to example 1 fig.2a) shows the defeat relations between the union of $\Delta_{d}$ and $\Delta_{r}$ 's arguments (where no partial ordering on the arguments is given). A unidirectional arrow indicates that the argument at the tail defeats the argument at the head of the arrow. A bidirectional arrow indicates a symmetric defeat. Note that $A 3$ rebut defeats $B 2$, but not vice versa, as $A 3$ also undercut defeats $B 2$ (see [12]).

The final stage in defining an argumentation system is to determine which arguments are preferred on the basis of the ways in which they interact. We employ Dung's seminal 'calculus of opposition' [4] to determine the preferred arguments from an argumentation framework (Args, Defeat). Firstly, we give Dung's definition of a preferred extension:

Definition 5. Let $A F$ be an argumentation framework (Args, Defeat). Then for any set $S \subseteq$ Args:

- $S$ is conflict free iff no argument in $S$ is defeated by an argument in $S$.

- An argument $A$ is acceptable w.r.t. $S$ iff each argument defeating $A$ is defeated by an argument in $S$.

- A conflict free set of arguments $S$ is admissible iff each argument in $S$ is acceptable with respect to $S$.

- A conflict free set of arguments $S$ is a preferred extension iff it is a maximal (w.r.t. set inclusion) admissible set.

Definition 6. Let $S_{1}, \ldots, S_{1}$ be the set of all preferred extensions of (Args, Defeat). Then $\bigcap_{i=1}^{n} S_{i}$ is the set of preferred arguments of $A F$

Referring to e.g 1, the preferred extensions are $\{A 1, A 2, A 3\}$ and $\{B 1, B 2\}$, and so there are no preferred arguments. It is the role of the mediator agent (MA) to assign a partial ordering on the arguments in order to decide a preferred set of arguments. The MA can valuate (and thus order) arguments on the basis of case based reasoning and agents' reputations (see [16]). An example of the latter is when the hospital represented by $R A$ has performed several unsuccessful lung transplants from donors with a smoking history who did not have COPD. The mediator can use this information to now prioritise $d 4$ over $r 5$, and hence for $i=1 \ldots 3: A i \succ B 1$ and defeat $(A i, B 1)$ (see fig.2b). Hence, $A 1, A 2$ and $A 3$ will be preferred arguments and the organ will be labelled as non-viable.

\subsection{Use of Argument Schemes and the Role of the Mediator Agent}

The defeasible rules in eg. 1 can be described in terms of argument schemes, and associated critical questions [18] that help identify arguments for attacking these schemes.

\footnotetext{
${ }^{1}$ Also, it seems quite reasonable to us that a rule or its negation is the claim of an argument - "I would argue that if $\mathrm{X}$ and $\mathrm{Y}$ are the case then $\mathrm{Z}$ is the case".
} 
Rules $d 4$ and $d 5$ formalise the non-viability scheme:

(1) If $D$ is donor of organ $O(2)$ and $D$ has property $C$ (3) and $C$ is a contraindication for donating organ $O(4)$ Then organ $O$ is non-viable.

and a critical question for this scheme - Is it the case that $C$ is a contraindication for donating organ $O$ ? - is addressed by formalisation of $r 5$. Conceptualisation of argumentation knowledge in terms of schemes and critical questions provided a useful means for eliciting the required knowledge from doctors. At present we have upwards of thirty schemes and questions [15]. We give another example below.

Example 2. Let $r$ be a potential recipient for the donor $d$ 's kidney $k$. Let sve denote streptococcus viridans endocarditis, svi denote streptococcus iridans infection, $r_{-} p$ denote 'recipient property', and results_r_p denote 'results in recipient property'. Suppose the DA's knowledge base $\Delta_{d}$ containing rules formalising the non-viability scheme:

(1)If $D$ is donor of organ $O$ (2) and D has property $P 1$ (3) and property $P 1$ will result in a recipient having property $P 2$ which is a contraindication for donating $O$ (4) Then organ $O$ is non-viable.

$\mathrm{d} 1=d_{-} o(d, k), \mathrm{d} 2=d_{-} p(d, s v e), \mathrm{d} 3=d_{-} o(D, k) \wedge d_{-} p(D, s v e) \Rightarrow$ results_r_p$(R, s v i)$, $\mathrm{d} 4=d_{-} o(D, k) \wedge$ results_r$r_{-} p(R, s v i) \Rightarrow \operatorname{contra}(D, k), \mathrm{d} 5=\operatorname{contra}(D, O) \Rightarrow \neg v$ $(D, O)$

The recipient agent's knowledge base $\Delta_{r}$ contains $\mathrm{r} 1=d_{-} o(d, k)$ and $\mathrm{r} 2=r_{-} p(R, s v i) \Rightarrow \neg\left(d_{-} o(D, k) \wedge\right.$ results_r_p$(R, s v i) \Rightarrow$ contra $\left.(D, k)\right)$ $\mathrm{r} 3=\operatorname{urgency}(R, 0) \Rightarrow \neg\left(d_{-} o(D, k) \wedge\right.$ results_r_p$\left.(R, \operatorname{svi}) \Rightarrow \operatorname{contra}(D, k)\right)$ $\mathrm{r} 4=$ plan_action $(R$, penicillin $) \Rightarrow \neg$ results_r_p $(R$, svi $)$ r6 $=d \_o(D, O) \wedge \operatorname{match}(D, R) \wedge \sim \operatorname{contra}(R, O) \Rightarrow v(D, O)$

Rules $\mathrm{r} 2$ and $\mathrm{r} 3$ are used to construct arguments addressing the critical question 'Is it the case that a transplant resulting in recipient having property $P 2$ is a contraindication for donating $O$ ?'. In particular $\mathrm{r} 2$ argues that if a recipient already has streptococcus viridans infection then it is not a contraindication, and $\mathrm{r} 3$ argues that if a recipient is at maximum urgency level 0 then it is not a contraindication. Rule $\mathrm{r} 4$ is used to construct an argument addressing the critical question 'Can the recipient be prevented from having property $P 2$ ', by positing administration of prophylactic penicillin.

We conclude with some remarks on the role of the mediator agent (MA). As discussed in eg 1 the MA may assign an ordering to arguments on the basis of an agent's reputation. As well as assigning an ordering to arguments, an MA can construct new arguments to decide a preference. To illustrate, consider e.g.2 in which the arguments constructed will interact in the same way as in e.g 1 . DA can construct an argument $A 3$ for nonviable based on a donor's medical condition resulting in a recipient being infected, as well as $A 1$ based on rule d4, and $A 2$ based on d3,d4. $A 1, A 2$ and $A 3$ rebut defeat and are rebut defeated by an RA argument $B 1$ that the recipient is already infected (based on rule r2). $A 2$ and $A 3$ undercut defeat RA's argument $B 2$ for viability based on r6. We thus obtain the same framework shown in fig.2a). In certain cases the issue may be more properly resolved not on the basis of whether RA or DA's arguments are stronger, but rather on the basis of legislation specific to the area of jurisdiction represented by the donor organisation. For example, in the case where a donor has the HIV virus and a recipient is already infected with the HIV virus, legislation may vary as to whether 
a transplant in such circumstances is legal or not. This legislation can be encoded for use by the MA to construct arguments to resolve the issue. For example, an argument $C 1$ that is built from the rule recipient_jurisdiction $(R, \operatorname{spain}) \Rightarrow \neg\left(r_{-} p(R, h i v)\right.$ $\Rightarrow \neg\left(d_{-} o(D, O) \wedge\right.$ results_r $\left.\left.r_{-} p(R, h i v) \Rightarrow \operatorname{contra}(D, O)\right)\right)$ will be stronger (given that legislative arguments are given highest priority) and so asymmetrically defeat $B 1$. Consequently, $C 1, A 1, A 2$ and $A 3$ will now be preferred.

\section{Conclusions and Future Work}

In this paper we have described an extension to the CARREL agent-based organization. The extension describes a novel application of argumentation theory in that we have formalised a framework for agent argumentation over the viability of organs for transplantation. We believe that our approach solves complex problems in the transplantation domain through efficient exchange of information and argumentation based reasoning over this information to support decision making. In particular, we believe our work has the potential to increase the number of human organs that current selection and assignation processes make available for transplantation, and thus reduce the growing disparity between supply of, and demand for, organs. Of further benefit is that a record of the agents' argumentation provides an explanatory audit trail for future reference by medical researchers, as well as a justification (that may be required in a legal context) for what are often potentially life threatening decisions.

The argumentation framework described in this paper differs from related formalisms [12,6] in that it allows for rules and their negations to be the claims of arguments, enabling formulation of Pollock's style defeats [11] as rebut rather than the standard undercut defeats. Note that future work will allow for use of strict rules in the construction of arguments; complications arising from their use (see [2]) have motivated the restriction to defeasible rules in the work presented here. Evaluation of the preferred status of arguments may require that a mediator agent assign a partial ordering on arguments, or indeed construct further arguments. An immediate goal for future work is to formalise our proposals described in [16] for the mediator agent's use of Case-Based Reasoning, an 'Acceptability Criteria Knowledge Base' and agent reputations in the evaluation phase. Note that we have described a process whereby DA and RA arguments are submitted to the mediator without an option for making further counter-arguments in response. For example, suppose the DA and RA having submitted arguments as described in e.g. 1 Assuming that the DA has received information about the location of the potential recipient, it may instantiate and submit another argument for non-viability on the grounds that there are logistical contraindications given the RA's location and the organ's ischaemia time. This further 'round of argumentation' suggests formalisation in terms of an argumentation based dialogue. Indeed, there is a considerable body of work on formalising multi-agent argumentation based persuasion dialogues (e.g.,[10]) in which one agent attempts to persuade the other of the validity of its claim though multiple exchanges of attacking arguments. Recent work on persuasion dialogues also illustrates persuasion over action (e.g.,[1]) rather than beliefs. In this work, an argument scheme for action and its associated critical questions are used as the basis for definition of a dialogue protocol. An argument for action instantiating 
the proposed scheme can be attacked by arguments identified by critical questions such as: does the action have an undesirable side-effect? and is there an alternative action that realises the same goal? In future work we will need to consider such argumentation over actions given that donor and recipient agents submit arguments referencing planned actions that result in some world state supporting their claim for viability (as described in e.g. 2]

Acknowledgments. This paper was supported in part by the Grant FP6-IST-002307 (ASPIC). Thanks also to H. Prakken for useful discussion on the content of this paper.

\section{References}

1. K. M. Atkinson, T. J. M. Bench-Capon, and P. McBurney. A dialogue game protocol for multi-agent argument for proposals over action. In Proc. First International Workshop on Argumentation in Multi-Agent Systems (ArgMAS 2004), 2004.

2. M. Caminada and L. Amgoud. An axiomatic account of formal argumentation. In Proceedings of the AAAI-2005, 2005.

3. U. Cortés, J. Vázquez-Salceda, A. López-Navidad, and F. Caballero. UCTx: a multi-agent approach to model a transplant coordination unit. In Proceedings of the 3rd. Congrés Català dIntelligència Artificial, 2000.

4. P. M. Dung. On the acceptability of arguments and its fundamental role in nonmonotonic reasoning, logic programming and $n$-person games. Artificial Intelligence, 77:321-357, 1995.

5. M. Esteva, D. de la Cruz, and C. Sierra. Islander: an electronic institutions editor. In AAMAS. ACM, 2002.

6. A.J. García and G.R. Simari. Defeasible logic programming: an argumentative approach. Theory and Practice of Logic Programming, 4(1):95-138, 2004.

7. A. López-Navidad and F. Caballero. Extended criteria for organ acceptance: Strategies for achieving organ safety and for increasing organ pool. Clin Transplant, Blackwell Munksgaard, 17:308-324, 2003.

8. OCATT. Organització Catalana de Transplantaments (OCATT). http://www10.gencat.net/catsalut/ocatt/en/htm/index.htm.

9. ONT. Organización Nacional de Transplantes. http://www.msc.es/ont.

10. S. Parsons, M. Wooldridge, and L. Amgoud. On the outcomes of formal inter-agent dialogues. In Proceedings of the Second International Joint Conference on Autonomous Agents and Multi-Agent Systems (AAMAS 2003), 2003.

11. J. L. Pollock. Defeasible reasoning. Cognitive Science, 11:481-518, 1987.

12. H. Prakken and G. Sartor. Argument-based extended logic programming with defeasible priorities. Journal of Applied Non-Classical Logics, 7:25-75, 1997.

13. H. Prakken and G. Vreeswijk. Handbook of Philosophical Logic, second edition, chapter Logics for Defeasible Argumentation. Kluwer Academic Publishers, 2002.

14. G.R. Simari and R.P. Loui. A mathematical treatment of defeasible reasoning and its implementation. Artificial Intelligence, 53:125-157, 1992.

15. P. Tolchinsky and U. Cortés. Argument Schemes and Critical Questions for deciding upon the Viability of a Human Organ for transplantation. Technical report, Technical University Of Catalonia, 2005. http://www.lsi.upc.edu/ tolchinsky/sch-list.pdf. 
16. P. Tolchinsky, U. Cortés, J.C. Nieves, F. Caballero, and A. López-Navidad. Using arguing agents to increase the human organ pool for transplantation. In 3rd Workshop on Agents Applied in Health Care (IJCAI-05), 2005.

17. J. Vázquez-Salceda, U. Cortés, J. Padget, A. López-Navidad, and F. Caballero. The organ allocation process: a natural extension of the CARREL Agent-Mediated Electronic Institution. AiCommunications. The European Journal on Artificial Intelligence, 3(16), 2003.

18. D. N. Walton. Argumentation Schemes for Presumptive Reasoning. Lawrence Erlbaum Associates, Mahwah, NJ, USA, 1996. 